\title{
Rapports de la directrice exécutive
}

FORCAST (http ://forcast.forest.ca) a pour mission de promouvoir la coordination et l'orientation des sciences et technologies forestières en fonction des priorités et des objectifs nationaux, provinciaux et locaux. FORCAST a tenu un forum à Edmonton en octobre dernier, qui regroupé les individus et les organisations du secteur forestier qui ont un intérêt pour la science et la technologie. La rencontre a fait état des réalisations complétées depuis la mise en place du Plan d'action national en sciences et technologies forestières en 1998 (consultez le site Internet de FORCAST); qui a fait quoi et quelles sont les lacunes en science et technologie au Canada. Plusieurs réalisations seront publiées au début de 2001. Ceux qui participé à la réunion ont identifié les lacunes particulièrement en science et technologie au niveau des communications, du réseautage et des investissements.

Les communications au sein de notre secteur sont évidemment importantes, pour nous permettre d'apprendre, d'adapter et de gérer effectivement. Tout spécialement les communications avec les autres secteurs et les chefs de file gouvernementaux. Le ministre Goodale a déclaré dans son message vidéo présenté aux participants du forum, « Je veux être en mesure de dire au monde entier, en pleine confiance, que le Canada est le " meilleur intendant, promoteur, utilisateur et exportateur forestier du monde - celui qui est le plus à la fine pointe technologique, le plus soucieux de l'environnement, le plus responsable au niveau social, le plus productif et le plus compétitif - étant à tête du monde en tant que modèle vivant d'un développement durable réussi. » Il a ajouté « Nous devons également mieux informer tous les Canadiens - ainsi que les principaux preneurs de décision au niveau gouvernemental - que notre pays doit mettre en priorité l'accent sur la recherche et le développement, les sciences et les technologies touchant le secteur forestier. Autant les Canadiens savent que l'Internet, le commerce électronique, l'informatique et les technologies d'information sont essentiels, autant cela doit comprendre les sciences et technologies forestières tant à l'échelle nationale qu'internationale, nécessitant un travail cérébral plus intensif et les investissements (tant publics que privés) pour que le travail soit accompli. »

En 1998, l'orientation des sciences et technologies indiquait qu' " un élément qui avait empêché l'intégration des facteurs socio-économiques en aménagement forestier tels qu'identifié lors du Forum national sur les sciences et technologies forestières résidant dans le fait que la recherche utile n'a pas pu suivre l'évolution des valeurs sociales et des politiques forestières. Il en résulte que les inventaires forestiers et les collectes de données traditionnelles ne sont pas conçus pour recueillir ce genre d'information, et que la méthodologie et les approches sont loin d'être aussi avancées que, par exemple, les études de croissance et de rendement. »

En d'autres termes, le progrès dans ce domaine a été inadéquat au cours des deux dernières années, même si des processus valables de participation du public ont été incorporés dans plusieurs politiques provinciales et industrielles. Ces processus impliquent le public dans la prise de décision en aménagement forestier. Toutefois, les processus dans leur ensemble ne sont pas évalués pour leur efficacité et dans plusieurs cas, ce type de recherche n'est pas évalué ou disséminé. Les participants de l'atelier ont identifié ce domaine comme étant sous-évalué et sous-financé.
Le ministre Goodale a aussi déclaré « Ensemble, nous devons travailler plus fort et plus efficacement à rassembler des informations précises - complètes et précises - pour le public averti sur les pratiques d'aménagement forestier durable au Canada - qui comprendraient, et c'est probablement le point important, nos travaux collectifs sur la scène scientifique ».

En tant que praticiens forestiers, nous devons être plus accessible face au public et participer à l'accroissement de la sensibilisation sur l'importance économique, sociale et culturelle des forêts. Au sein de l'Institut, nous pouvons communiquer à nos membres l'importance d'une participation active du public dans les processus de présentation et de prises de décision (voir le mémoire de l'Institut sur la participation du public dans les processus de prise de décision dans le cas des forêts (Sept./Oct. 1998 pg 747-749). Nous pouvons aider le public à comprendre les processus de participation publique. Nous pouvons également faciliter le dialogue avec ces communautés, et faire le lien en reconnaissant la multitude d'utilisation des forêts. Au cours de ces activités, nous devrions enregistrer et recueillir les informations sur les retombées des processus et des dialogues, et entreposer ces données dans une banque centrale de telle sorte que les autres pourront apprendre et profiter de ces expériences.

Nous devrions agir selon le message du ministre Goodale et encourager les chefs de file politiques à mettre plus de ressources dans les recherches en foresterie sociale et les mettre au défi de mettre en tête de priorité les sciences et technologies ainsi la recherche et le développement en foresterie.

Nous devrions tous être fiers de démontrer avec le ministre Goodale que nous sommes les intendants forestiers les plus responsables, les plus innovateurs et les meilleurs de ce monde.

\footnotetext{
Executive Director

industry policies. These processes involve the public in forest management decision-making. However, the processes overall are not evaluated for their effectiveness and, in many cases, this type of research is not assessed or disseminated. The workshop participants identified this area as under valued and insufficiently funded.

Minister Goodale also stated "We, together, need to work much harder and much more effectively at putting accurate information - complete and accurate information - before a discerning public about Canada's sustainable forest management practices - including, and perhaps most importantly, our collective work on the scientific front."

We, as forest practitioners, need to be more accessible to the public and assist in building awareness of the importance of forests economically, socially and culturally. Within the CIF/IFC, we can communicate to our members the importance of active participation of the public in participation processes and decisionmaking (see CIF/IFC Position paper on Public Participation in decision-making about forests (Sept/Oct 1998, pp. 747-749). We can assist the public in understanding the public participation process. We can also facilitate dialogue with communities, and liaise in recognizing the wide variety of forest uses. In these activities, we should record and collect the information on $\mathbf{\$ 9 7 2}$
} 